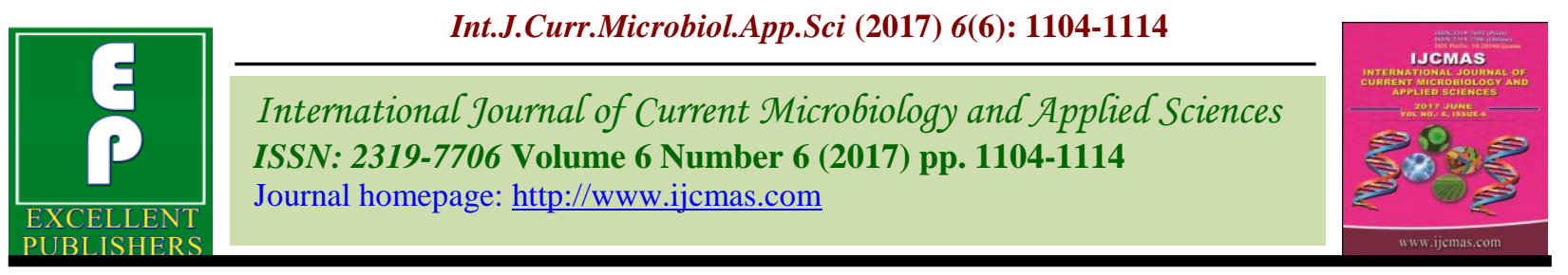

Original Research Article

https://doi.org/10.20546/ijcmas.2017.606.128

\title{
Physiological Variability in Streptomyces spp. A Causal Organism of Common Scab of Potato (Solanum tuberosum L.)
}

\author{
Rohit Badaya* and J.S. Srivastava \\ Department of Mycology and Plant Pathology, Institute of Agricultural Sciences, \\ Banaras Hindu University, Varanasi- 221005 (U.P.), India \\ *Corresponding author
}

\section{A B S T R A C T}

\section{Keywords}

Pathogenicity, Physiological Variability, Potato, Streptomyces Scabies.

Article Info

Accepted:

17 May 2017

Available Online:

10 June 2017
Common scab is an important disease of potato caused by Streptomyces scabies and other closely related species. Twenty seven cultures from different regions of Varanasi district were isolated and tested for their pathogenicity, using the variety Kufri Chandramukhi. Only Thirteen isolates were able to cause disease on potato tubers. After purification these cultures were named as S1, S2, S3, S4, S5, S6, S7, S8, S9, S10, S11, S12 and $\mathrm{S} 13$, then examined for their morphological, cultural and physiological properties so as to identify them with the help of available literature.

\section{Introduction}

Potato suffers from a number of diseases of varied origins, which are responsible for decrease in yield and its quality. Among the bacterial disease of potato, Common scab of potatoes is caused by Streptomyces scabies, a very prevalent, soil-inhabiting bacterium. This serious disease can be found in all potato- growing areas throughout the world. The scab organism sometimes occurs in soils where potatoes have never been grown. In most potato soils, however, scab was probably introduced with infected seed tubers. The major loss from common scab is lower market quality because tubers are unsightly or disfigured and have poor customer appeal. Where scab is severe, yields also may be reduced. The disease causes an annual loss of several million dollars in the United States. Common scab of potato, which was previously considered to be a minor disease, has created a serious threat to the potato cultivation due to increase scab incidence year after year. Due to its detrimental effect on appearance, grade and marketable yield, common scab of potato has been considered a disease of major economic importance (King et al., 1992). Common scab is now becoming a major problem in almost all the agroclimatic zones of India (Nagaich, 1983). Decrease in yield due to severe infections has been reported, but usually the grade quality of the tubers is affected. In addition, typical earthy odour of Streptomyces produced by geosmin, can make the flesh inedible (Lechevalier, 1988). 
Several species of Streptomyces are known to induce common scab. S. scabiei (formerly $S$. scabies) is described as the main causal agent, but it has been considered for several years as a species Incertae sedis. The taxonomy of this bacterium has been largely clarified by Lambert and Loria (1989a), who redefined the species using morphological (smooth gray spores borne in spiral chains) and physiological characteristics [production of melanin, utilization of the nine diagnostic sugars (L-arabinose, D-fructose, D-glucose, D-mannitol, inositol, raffinose, rhamnose, sucrose, and D-xylose)] recommended in the International Streptomyces Projects (Shirling and Gottlieb, 1966). Acid-tolerant strains of Streptomyces were also isolated from lesions of common scab (Bonde and Mclntyre, 1968). Lambert and Loria (1989b) flamed these strains $S$. acidiscabies and characterized them on the basis of morphological and physiological characteristics (Lambert and Loria, 1989b) and DNA-DNA homology (Healy and Lambert, 1991).

Careful and thorough characterization of pathogens and their pathogenicity and virulence-related genes is a prerequisite to the development of any successful control strategy. The aim of this work was to assess the physiological variability of Streptomyces spp. causing common scab of potato in Varanasi district (eastern U.P.).

\section{Materials and Methods}

\section{Isolation of the pathogen}

Isolation was made by following the techniques of Lawrence (1956) and Loria and Davis (1989). Potato tubers affected by common scab (Normal and Pitted scab) were collected from several potato growing areas of Varanasi. Fresh diseased tubers collected from various places were washed under tap water to remove adhering soil particles. After washing, tubers were soaked in a mixture of phenol and sterile water (1:140) for 10 minutes to eliminate the bacterial contamination and then rinsed 3-4 times with sterile distilled water for 15 minutes. Then, a slice was cut with sterilized scalpel to remove the scabbed areas along with immediately underlying healthy tissues. Approximately, five grams peeling from scabby tuber was macerated in $20 \mathrm{ml}$ sterile distilled water with a pestle and mortar and volume was then maintained $100 \mathrm{ml}$. The mixture was left for few minutes and then $0.1 \mathrm{ml}$ of this suspension was mixed with $20 \mathrm{ml}$ of molten medium (Czapek-dox medium) at $45^{\circ} \mathrm{C}$ and poured into sterilized petridishes. Plates were then incubated at $28^{\circ} \mathrm{C} \pm 1$ for 6-7 days. Later colonies were transferred to yeast extract malt extract agar medium.

\section{Test of Pathogenicity}

\section{Pot method}

To test the pathogenicity in pots, the method described by Labruyere (1971) and Loria and Davis (1989) was followed. Inoculum for pathogenicity test was produced on YME Agar medium. Contents from two plates were thoroughly with autoclaved soil (a mixture of sandy soil + compost in the ratio 4:1). Potting mixture was then placed in earthen pots $(20 \times$ $15 \mathrm{~cm}$ in size). Certified seed tubers of the variety $\mathrm{KCM}$ which were free from scab lesions were surface disinfected with sodium hypochlorite $(0.5 \%)$ solution for 10 minutes and then planted @ 3 tubers per pot, approximately $5 \mathrm{~cm}$ below the surface of the potting mixture and then placed in the wire net house. Pots were irrigated with tap water on 20th day, thereafter; irrigation was kept to a minimum in order to facilitate the infection of newly enlarging tubers. After 10 weeks, tubers were harvested and washed in running water and evaluated for scab occurrence. 


\section{Characterization of Streptomyces isolates}

\section{Morphological studies}

\section{Aerial mass colour}

Each isolate was cultured for 10-14 days in the dark at $30{ }^{\circ} \mathrm{C}$ on different media. The colour of the mycelium and spores was recorded in bright sun light, and isolates were classified according to the Tresner-Backus colour wheel (Red, Yellow, Green, Blue, Violet, Grey or White) on different media (Shirling and Gottlieb, 1966).

\section{Colour of the substrate mycelium}

Colour of the substrate mycelium was determined by observing reverse (under) side of mass growth on various media in petri dishes. Cultures were assigned to the following colour groups (Szabo and Marton, 1964):-

Brown, Grey, Yellow, Yellow-brown

\section{Observation to see the fragmentation of substrate mycelium}

For observing the fragmentation of substrate mycelium, thin vertical blocks of agar was cut from 7 days old culture growing on glycerol asparagine agar medium and viewed by placing it on a slide under cover slip by light microscope (Cross, 1986).

In another study, spore chain and sporophore morphology was determined by observing the mycelium and spore chains adhered to the cover slip inserted obliqually in preinoculated medium in petriplates. For detail studies, cover slips were withdrawn from the petridish after 15 days of incubation and mounted upper surface down in water containing wetting agent. Cover slips along with adhering spore chain mycelia were examined under light microscope. Types of spore chain of each isolate were determined and characterized according to the procedure described by Shirling and Gottlieb (1966). The form of spore chain was determined as flexuous or spirals.

\section{Cultural studies}

The following culture media were used for cultural studies:-

Yeast Extract Malt Extract Agar medium (YME)

Inorganic Salt Starch Agar medium

Glycerol Asparagine Agar medium (GA)

Sucrose Nitrate Agar medium

Nutrient Agar medium

Six plates of each medium for each culture were prepared and inoculated in cross hatched pattern. Plates were incubated at $28 \pm 10 \mathrm{C}$ in an incubator. After 14 days of incubation, surface of the cultured test organism was examined directly under microscope. Growth of vegetative mycelium (VM) as well as aerial mycelium (AM) was as- Abundant/Moderate/ Scanty (A/M/S). Colony texture of aerial mycelium (AM) was characterized asCottony/Granular $(\mathrm{C} / \mathrm{G})$ and Colour of aerial mycelium (AM) was characterized asGrey/White/Yellow/Brown (G/W/Y/B).

\section{Physiological studies}

\section{Carbon utilization test}

The Carbon utilization medium which was given by Shirling and Gottlieb (1966) was used for this test. Commercially available pure carbon sources certified as free from admixture with any other carbohydrates were 
used for this study. Carbon sources used for this test were as follows:

Fructose L-Arabinose

Maltose Raffinose

Sucrose Galactose

D-Xylose Inositol (meso)

Mannitol Rhamnose

Glucose (Used for positive Control)

Carbon sources were sterilized aseptically by using Millipore membrane filters (pore size $0.22 \mu \mathrm{m}) .10 \%$ solution in distilled water (W/V) was used for sterilization. Separate syringe and adoptors were used for each sugar/carbohydrate. One $\mathrm{ml}$ of Pridham and Gottlieb trace salt solution (Pridham and Gottlieb, 1948) was added per liter to the basal mineral salt medium.

After autoclaving, flasks containing basal medium were allowed to cool at 600C. Different carbon source were added separately in each flask @ 100ml/1. of basal medium as to ensure 1\% final concentration of carbon source in the medium.

Mixture was agitated thoroughly and poured into petridishes $(25 \mathrm{ml}$ medium/plate $)$. Duplicate plates of each carbon source for each culture were streaked and incubated at $28^{\circ} \mathrm{C} \pm 1{ }^{\circ} \mathrm{C}$ in an incubator and the results were recorded.

\section{Sensitivity to antibiotics and crystal violet}

Sensitivity against antibiotics and crystal violet was judged on yeast extract malt extract agar medium. Two antibiotics (streptomycin sulphate and penicillin G) and one inhibitory chemical (crystal violet) were used for this purpose. Resistance against antibiotics or crystal violet was defined as the ability of an isolate to grow on YME supplemented with $20 \mathrm{mg} / \mathrm{l}$ of streptomycin sulphate, $1000 \mathrm{IU} / \mathrm{I}$ of penicillin $\mathrm{G}$ or $0.56 \mathrm{mg} / \mathrm{l}$ of crystal violet, respectively.

Plates containing above chemicals were streaked (in duplicate) with the culture to be tested separately and incubated at $28^{\circ} \mathrm{C}$ for 7 days. Growth of the test culture was recorded as positive and otherwise negative.

\section{Test of sensitivity to $\mathrm{pH}$}

Growth of the test culture was assessed on YME medium at five different $\mathrm{pH}$ levels i.e. $4,5,6,7$ and 8 for this test. $\mathrm{PH}$ of the medium was adjusted by adding $0.1 \mathrm{~N} \mathrm{HCl}$ or $0.1 \mathrm{~N}$ $\mathrm{NaOH}$.

Plates were incubated at $280 \mathrm{C}$ for 7 days and the presence or absence of the growth was recorded.

\section{Melanin production}

Melanin production was determined after 4 days of inoculation on peptone yeast extract iron agar and tyrosine agar medium (Shirling and Gottlieb, 1966). Heavy inoculum of spores and aerial mycelium was picked up from 2 weeks old culture of the test pathogen and streaked on to the surface of agar slants. Observation, regarding melanin production was done.

Comparison was made between inoculated and uninoculated slants. Cultures forming greenish brown to brown black diffusible pigment or a distinct brown pigment or modified by any other colour was recorded as positive (+). Absence of brown to black colour or total absence of diffusible pigment was recorded as negative (-).

Nitrate reductase activity, starch hydrolysis, gelatin liquification, coagulation and peptonization of milk were done as given by Kiraly (1974). 


\section{Results and Discussion}

\section{Characterization of the pathogen morphological characteristics}

On the basis of morphological properties exhibited on YME Agar medium, The thirteen pathogenic cultures were grouped into two categories i.e. spiral spore chain types, viz. S1, S2, S4, S8, S9, S10, S11 and S13 and flexuous spore chain types, viz. S3, S5, S6, S7 and S12. All the spiral types' beared cylindrical spores formed on long chains whereas flexuous isolates contained oval spores formed in medium to long chains (Table 1).

\section{Cultural characteristics}

All the isolates produced abundant aerial mycelium (AM) and vegetative mycelium (VM) on glycerol asparagine agar medium. Colony texture was cottony in spiral isolates and granular in flexuous type (Table 2). There was no significant difference in cultural characteristics of the isolates growing on either glycerol asparagine agar (GA) medium or starch agar medium. All the isolates produced abundant vegetative mycelium and showed scanty aerial growth on nutrient agar medium. Cultural characteristics on yeast extract malt extract agar medium (YME) was almost similar with glycerol asparagine agar medium (GA). Production of moderate vegetative mycelium was observed on sucrose nitrate agar medium in all the cultures except S8 and S13 where it was abundant, whereas scanty aerial growth was observed with all the cultures except S6, S8 and S13.

\section{Physiological characteristics}

All the cultures were examined for their physiological properties as exhibited in various diagnostic tests (Table 3). Utilization of various sugars by the isolates was one of the diagnostic tests and it was found that most of the cultures grew well in glucose, fructose, maltose, xylose, mannitol and galactose except isolates S3 and S7 which preferred glucose and maltose only. Sucrose was not utilized effectively by the isolates S3, S6 and S7. Rest of the isolates utilized the same. Arabinose was utilized by most of the isolates except S3, S4, S6 and S7. Raffinose was utilized by most of the isolates except $\mathrm{S} 3, \mathrm{~S} 4$, S5, S7, S9 and S12. All the isolates utilized inositol except S3, S7 and S12. None of the isolates was able to grow in presence of streptomycin $(20 \mu \mathrm{g} / \mathrm{ml})$. However, the isolates $\mathrm{S} 2, \mathrm{~S} 3, \mathrm{~S} 5, \mathrm{~S} 6$ and $\mathrm{S} 11$ were resistant to penicillin $\mathrm{G}(100 \mu \mathrm{g} / \mathrm{ml})$.

Peptonization of milk was observed with the isolates $\mathrm{S} 1, \mathrm{~S} 7, \mathrm{~S} 8$ and $\mathrm{S} 12$ whereas isolates S3, S5 and S9 exhibited slow peptonization. Rest of the isolates showed negative reaction. Similarly, coagulation of milk was observed in isolates S1, S3, S11 and S12 whereas isolate $\mathrm{S} 7$ exhibited slow coagulation. Rest of the isolates showed negative reaction. Surface ring colour of the isolates S1, S2, S4, S10, S11 and S13 was either grey or brown whereas in the remaining isolates, ring was whitish or yellowish. All the isolates were $\mathrm{H} 2 \mathrm{~S}$ negative and starch hydrolysis positive. Isolates S4, S8, S9, S10 and S13 produced melanin pigment in both organic (peptone yeast extract iron agar medium) and inorganic (Tyrosine agar) medium. Isolate S1 produced melanin only when grown on inorganic medium. Rest of the isolates did not produce melanin in any of the two media.

The isolates S10 and S12 were resistant to crystal violet $(0.5 \mu \mathrm{g} / \mathrm{ml})$. The isolates $\mathrm{S} 6$, S11, S12 and S13 were resistant to phenol $(0.1 \%)$ while the remaining isolates were sensitive. None of the cultures were able to grow at $\mathrm{pH}$ 4.5. Gelatin liquification was observed with all the isolates either slow or fast except S2, S3, S5, S6, S7 and S10 which showed negative reaction. Nitrate reductase test was found positive with all the isolates. 
Table.1 Morphological characteristics of the isolates of Streptomyces spp

\begin{tabular}{|c|c|c|c|c|c|c|c|c|c|c|c|c|c|}
\hline \multirow{2}{*}{$\begin{array}{l}\text { Morphological } \\
\text { Characteristics }\end{array}$} & \multicolumn{13}{|c|}{ Isolates } \\
\hline & $\mathbf{S}_{1}$ & $\mathbf{S}_{2}$ & $\mathbf{S}_{\mathbf{3}}$ & $\mathbf{S}_{4}$ & $\mathbf{S}_{5}$ & $S_{6}$ & $\mathbf{S}_{7}$ & $\mathbf{S}_{8}$ & $\mathbf{S}_{9}$ & $\mathbf{S}_{10}$ & $\mathbf{S}_{11}$ & $\mathrm{~S}_{12}$ & $\mathbf{S}_{13}$ \\
\hline $\begin{array}{l}\text { Colour of Aerial } \\
\text { Mycelium (Grey-G, } \\
\text { White-W, Yellow-Y) }\end{array}$ & $\mathrm{W}$ & $\mathrm{W}$ & $\mathrm{W}$ & $\mathrm{W}$ & $\mathrm{G}$ & $\mathrm{G}$ & $\mathrm{W}$ & $Y$ & $\mathrm{Y}$ & $\mathrm{G}$ & $G$ & $\mathrm{Y}$ & $\mathrm{W}$ \\
\hline $\begin{array}{l}\text { Colour of Substrate } \\
\text { Mycelium } \\
\text { (Yellow Brown-YB, } \\
\text { Brown-B, Yellow- } \\
\text { Y) }\end{array}$ & YB & YB & B & YB & $\mathrm{YB}$ & YB & B & $\mathrm{Y}$ & $\mathrm{Y}$ & B & YB & $Y$ & YB \\
\hline $\begin{array}{l}\text { Nature of Substrate } \\
\text { Mycelium } \\
\text { Non Fragmented-NF }\end{array}$ & $\mathrm{NF}$ & $\mathrm{NF}$ & $\mathrm{NF}$ & NF & NF & $\mathrm{NF}$ & $\mathrm{NF}$ & $\mathrm{NF}$ & $\mathrm{NF}$ & $\mathrm{NF}$ & $\mathrm{NF}$ & $\mathrm{NF}$ & $\mathrm{NF}$ \\
\hline $\begin{array}{l}\text { Branching of } \\
\text { sporophores } \\
\text { (Monopodial-MP) } \\
\end{array}$ & MP & MP & MP & MP & MP & $\mathrm{MP}$ & MP & MP & MP & MP & MP & MP & MP \\
\hline \begin{tabular}{|l|} 
Sporulation pattern \\
Or Spore chain \\
(Spiral- S, Flexuous- \\
F)
\end{tabular} & $S$ & $S$ & $\mathrm{~F}$ & $S$ & $\mathrm{~F}$ & $\mathrm{~F}$ & $\mathrm{~F}$ & $S$ & $S$ & $S$ & $S$ & $\mathrm{~F}$ & $S$ \\
\hline $\begin{array}{l}\text { Spore Shape } \\
\text { (Cylindrical- C, } \\
\text { Oval-O) } \\
\end{array}$ & $\mathrm{C}$ & $\mathrm{C}$ & $\mathrm{O}$ & $\mathrm{C}$ & $\mathrm{O}$ & $\mathrm{O}$ & $\mathrm{O}$ & $\mathrm{C}$ & $\mathrm{C}$ & $\mathrm{C}$ & $\mathrm{C}$ & $\mathrm{O}$ & $\mathrm{C}$ \\
\hline $\begin{array}{l}\text { Spore Size } \\
\text { (Width x Length, } \\
\mu \mathrm{m})\end{array}$ & $\begin{array}{c}0.8- \\
0.9 \\
\mathrm{X} \\
1.2- \\
1.4\end{array}$ & $\begin{array}{c}0.9- \\
1.0 \\
\mathrm{X} \\
1.3- \\
1.7\end{array}$ & $\begin{array}{c}0.7- \\
0.8 \\
\mathrm{X} \\
0.9- \\
1.0\end{array}$ & $\begin{array}{c}0.6- \\
0.9 \\
x \\
1.1- \\
1.5\end{array}$ & $\begin{array}{c}0.7- \\
0.9 \\
x \\
0.9- \\
1.0\end{array}$ & $\begin{array}{c}0.6- \\
0.9 \\
\mathrm{x} \\
1.0-1.1\end{array}$ & $\begin{array}{c}0.9- \\
1.0 \\
\mathrm{X} \\
0.9- \\
1.0\end{array}$ & $\begin{array}{c}0.7- \\
1.1 \\
\mathrm{X} \\
1.3- \\
1.7\end{array}$ & $\begin{array}{c}0.7- \\
1.0 \\
\mathrm{X} \\
1.4- \\
1.6\end{array}$ & $\begin{array}{c}0.8- \\
0.9 \\
\mathrm{X} \\
1.5- \\
1.7\end{array}$ & $\begin{array}{c}0.9- \\
1.0 \\
\mathrm{X} \\
1.1- \\
1.2\end{array}$ & $\begin{array}{c}0.7- \\
1.0 \\
\mathrm{X} \\
0.9- \\
1.1\end{array}$ & $\begin{array}{c}0.8- \\
1.0 \\
\mathrm{X} \\
1.2- \\
1.4\end{array}$ \\
\hline
\end{tabular}


Table.2 Cultural characteristics of the isolates of Streptomyces spp

\begin{tabular}{|c|c|c|c|c|c|c|c|c|c|c|c|c|c|c|}
\hline \multirow[t]{2}{*}{ Medium } & \multirow[t]{2}{*}{ Properties } & \multicolumn{13}{|c|}{ Isolates } \\
\hline & & $\mathbf{S}_{1}$ & $\mathbf{S}_{\mathbf{2}}$ & $\mathbf{S}_{\mathbf{3}}$ & $\mathbf{S}_{4}$ & $\mathbf{S}_{5}$ & $\mathbf{S}_{6}$ & $\mathbf{S}_{7}$ & $\mathbf{S}_{8}$ & $\mathbf{S}_{\mathbf{9}}$ & $\mathbf{S}_{10}$ & $\mathrm{~S}_{11}$ & $\mathbf{S}_{12}$ & $\overline{S_{13}}$ \\
\hline \multirow{4}{*}{$\begin{array}{l}\text { Glycerol } \\
\text { Asparagine } \\
\text { Agar } \\
\text { Medium }\end{array}$} & Vegetative mycelium (VM)- Abundant/ Moderate/ Scanty (A/M/S) & A & A & A & A & A & A & A & A & $\mathrm{A}$ & A & A & A & $\mathrm{A}$ \\
\hline & Aerial mycelium (AM)- Abundant/Moderate/ Scanty (A/M/S) & $\mathrm{A}$ & $\mathrm{A}$ & $\mathrm{A}$ & $\mathrm{A}$ & A & A & $\mathrm{A}$ & $\mathrm{A}$ & $\mathrm{A}$ & $\mathrm{A}$ & $\mathrm{A}$ & $\mathrm{A}$ & $\mathrm{A}$ \\
\hline & Aerial mycelium (AM)- Colony texture-cottony/ Granular (C/G) & $\mathrm{C}$ & $\mathrm{C}$ & $\mathrm{G}$ & $\mathrm{C}$ & G & G & $\mathrm{G}$ & $\mathrm{C}$ & $\mathrm{C}$ & $\mathrm{C}$ & $\mathrm{C}$ & $\mathrm{G}$ & $\mathrm{C}$ \\
\hline & Aerial mycelium (AM)- Colour-Grey/White/ Yellow/Brown(G/W/Y/B) & $\mathrm{G}$ & $\mathrm{G}$ & $\mathrm{W}$ & $\mathrm{G}$ & G & $\mathrm{Y}$ & $\mathrm{W}$ & $\mathrm{G}$ & $\mathrm{G}$ & W & $\mathrm{W}$ & $\mathrm{B}$ & $\mathrm{G}$ \\
\hline \multirow{4}{*}{\begin{tabular}{|l|} 
Starch \\
Agar \\
Medium
\end{tabular}} & Vegetative mycelium (VM)- Abundant/ Moderate/Scanty (A/M/S) & A & A & A & A & A & A & A & A & $\mathrm{A}$ & A & A & $\mathrm{A}$ & A \\
\hline & Aerial mycelium (AM)- Abundant/Moderate/ Scanty (A/M/S) & $\mathrm{A}$ & A & A & A & A & A & A & A & $\mathrm{A}$ & A & A & $\mathrm{A}$ & A \\
\hline & Aerial mycelium (AM)- Colony texture-cottony/ Granular (C/G) & $\mathrm{G}$ & $\mathrm{G}$ & $\mathrm{G}$ & $\mathrm{C}$ & G & $\mathrm{G}$ & $\mathrm{G}$ & $\mathrm{C}$ & $\mathrm{C}$ & $\mathrm{C}$ & $\mathrm{G}$ & $\mathrm{G}$ & $\mathrm{C}$ \\
\hline & $\begin{array}{l}\text { Aerial mycelium (AM)- Colour-Grey/White/ Yellow/Brown/Dark } \\
\text { (G/W/Y/B/D) }\end{array}$ & G & G & $\mathrm{W}$ & G & W & $\mathrm{W}$ & W & $\mathrm{W}$ & $\mathrm{G}$ & W & $\mathrm{G}$ & $\begin{array}{l}\mathrm{D} \\
\mathrm{G}\end{array}$ & $\mathrm{W}$ \\
\hline \multirow{4}{*}{\begin{tabular}{|l|} 
Nutrient \\
Agar \\
Medium
\end{tabular}} & $\begin{array}{l}\text { Vegetative mycelium (VM)- Abundant/ } \\
\text { Moderate/ Scanty (A/M/S) }\end{array}$ & $\mathrm{A}$ & A & $\mathrm{A}$ & $\mathrm{A}$ & A & $\mathrm{A}$ & $\mathrm{A}$ & $\mathrm{A}$ & $\mathrm{A}$ & $\mathrm{A}$ & $\mathrm{A}$ & $\mathrm{A}$ & $\bar{A}$ \\
\hline & Aerial mycelium (AM)- Abundant/Moderate/ Scanty (A/M/S) & $\mathrm{S}$ & $\mathrm{S}$ & $\mathrm{S}$ & $\mathrm{S}$ & $\mathrm{S}$ & $\mathrm{S}$ & $\mathrm{S}$ & $\mathrm{S}$ & $\mathrm{S}$ & $\mathrm{S}$ & $\mathrm{S}$ & $\mathrm{S}$ & $\mathrm{S}$ \\
\hline & Aerial mycelium (AM)- Colony texture-Cottony/ Granular (C/G)(- No growth) & - & - & - & - & - & - & - & - & - & - & - & - & - \\
\hline & $\begin{array}{l}\text { Aerial mycelium (AM)- Colour- Grey/White/ Yellow/Brown (G/W/Y/B) (- No } \\
\text { growth) }\end{array}$ & - & - & - & - & - & - & - & - & - & - & - & - & - \\
\hline \multirow{4}{*}{$\begin{array}{l}\text { Yeast } \\
\text { Extract } \\
\text { Malt } \\
\text { Extract } \\
\text { Agar }\end{array}$} & $\begin{array}{l}\text { Vegetative mycelium (VM)- Abundant/ } \\
\text { Moderate/Scanty (A/M/S) }\end{array}$ & A & A & A & M & A & A & A & A & M & A & A & M & A \\
\hline & Aerial mycelium (AM)- Abundant/Moderate/ Scanty (A/M/S) & A & A & A & A & A & A & A & A & $\mathrm{A}$ & A & A & A & A \\
\hline & Aerial mycelium (AM)- Colony texture-Cottony/ Granular (C/G) & $\mathrm{C}$ & $\mathrm{C}$ & $\mathrm{G}$ & $\mathrm{C}$ & G & $\mathrm{G}$ & $\mathrm{G}$ & $\mathrm{C}$ & $\mathrm{C}$ & $\mathrm{C}$ & $\mathrm{C}$ & $\mathrm{G}$ & $\mathrm{C}$ \\
\hline & $\begin{array}{l}\text { Aerial mycelium (AM)- Colour- Grey/White/ Yellow/Brown/Dark/Light } \\
\text { (G/W/Y/B/D/L) }\end{array}$ & $\mathrm{W}$ & $\mathrm{W}$ & $\mathrm{W}$ & $\mathrm{G}$ & LB & DG & $\mathrm{W}$ & $\mathrm{W}$ & $\mathrm{G}$ & W & $\mathrm{W}$ & $\mathrm{W}$ & $\mathrm{W}$ \\
\hline \multirow{4}{*}{$\begin{array}{l}\text { Sucrose } \\
\text { Nitrate } \\
\text { Agar }\end{array}$} & Vegetative mycelium (VM)- Abundant/Moderate/ Scanty (A/M/S) & $\mathrm{M}$ & $\mathrm{M}$ & $\mathrm{M}$ & $\mathrm{M}$ & M & $\mathrm{M}$ & M & A & $\mathrm{M}$ & $\mathrm{M}$ & $\mathrm{M}$ & $\mathrm{M}$ & A \\
\hline & Aerial mycelium (AM)- Abundant/Moderate/ Scanty (A/M/S) & $\mathrm{S}$ & $\mathrm{S}$ & $\mathrm{S}$ & $\mathrm{S}$ & $\mathrm{S}$ & A & $\mathrm{S}$ & A & $\mathrm{S}$ & $\mathrm{S}$ & $\mathrm{S}$ & $\mathrm{S}$ & A \\
\hline & Aerial mycelium (AM)- Colony texture-Cottony/ Granular (C/G) & $\mathrm{C}$ & $\mathrm{C}$ & $\mathrm{C}$ & $\mathrm{C}$ & G & G & $\mathrm{C}$ & $\mathrm{C}$ & $\mathrm{G}$ & $\mathrm{C}$ & $\mathrm{C}$ & $\mathrm{G}$ & $\mathrm{C}$ \\
\hline & Aerial mycelium (AM)- Colour- Grey/White/ Yellow/Brown (G/W/Y/B) & $\mathrm{W}$ & G & $\mathrm{W}$ & W & $\begin{array}{l}\text { W/ } \\
\mathrm{Y}\end{array}$ & $\mathrm{G}$ & $\mathrm{W}$ & G & $\mathrm{G}$ & $\mathrm{G}$ & DG & W & $\mathrm{W}$ \\
\hline
\end{tabular}


Table.3 Physiological characteristics of the isolates of Streptomyces spp

\begin{tabular}{|c|c|c|c|c|c|c|c|c|c|c|c|c|c|}
\hline \multirow[t]{2}{*}{ Utilization of Sugars } & \multicolumn{13}{|c|}{ Isolates } \\
\hline & $\mathbf{S}_{1}$ & $\mathbf{S}_{2}$ & $\mathbf{S}_{\mathbf{3}}$ & $\mathbf{S}_{4}$ & $\mathbf{S}_{5}$ & $S_{6}$ & $\mathbf{S}_{7}$ & $\mathbf{S}_{8}$ & $\mathbf{S}_{\mathbf{9}}$ & $\mathbf{S}_{10}$ & $S_{11}$ & $S_{12}$ & $\mathbf{S}_{13}$ \\
\hline Control & - & - & - & - & - & - & - & - & - & - & - & - & - \\
\hline Fructose & + & + & - & + & + & + & - & + & + & + & + & + & + \\
\hline Arabinose & + & + & - & - & + & - & - & + & + & + & + & + & + \\
\hline Sucrose & + & + & - & + & + & - & - & + & + & + & + & + & + \\
\hline Maltose & + & + & + & + & + & + & + & + & + & + & + & + & + \\
\hline Galactose & + & + & - & + & + & + & - & - & + & + & + & - & + \\
\hline Glucose & + & + & + & + & + & + & + & + & + & + & + & + & + \\
\hline Mannitol & + & + & - & + & + & + & - & + & + & + & + & + & + \\
\hline Xylose & + & + & - & + & + & + & - & - & + & + & + & + & + \\
\hline Raffinose & + & + & - & - & - & + & - & + & - & + & + & - & + \\
\hline Inositol & + & + & - & + & + & + & - & + & + & + & + & - & + \\
\hline \multicolumn{14}{|l|}{ Sensitivity to antibiotics } \\
\hline Streptomycin $(20 \mu \mathrm{g} / \mathrm{ml})$ & - & - & - & - & - & - & - & - & - & - & - & - & - \\
\hline Penicillin G $(100 \mu \mathrm{g} / \mathrm{ml})$ & - & + & + & - & + & + & - & - & - & - & + & - & - \\
\hline \multicolumn{14}{|l|}{ (- Sensitive, + Resistant) } \\
\hline \multicolumn{14}{|l|}{ Action on Milk } \\
\hline Peptonization & + & - & Slow & - & Slow & - & + & + & Slow & - & - & + & - \\
\hline Coagulation & + & - & + & - & - & - & Slow & - & - & - & + & + & - \\
\hline Surface ring colour & Brown & Grey & Yellow & Brown & Yellow & Yellow & Yellow & White & White & Grey & Grey & Yellow & Brown \\
\hline $\mathrm{H}_{2} \mathrm{~S}$ Production & - & - & - & - & - & - & - & - & - & - & - & - & - \\
\hline Change in pH & 8.02 & 8.01 & 7.15 & 6.67 & 7.53 & 7.08 & 6.53 & 7.25 & 7.79 & 7.3 & 6.78 & 7.93 & 8.09 \\
\hline \multicolumn{14}{|c|}{ Melanin Production on medium } \\
\hline $\begin{array}{l}\text { Peptone yeast extract iron } \\
\text { agar }\end{array}$ & - & - & - & + & - & - & - & + & + & + & - & - & + \\
\hline Tyrosine agar & + & - & - & + & - & - & - & + & + & + & - & - & + \\
\hline \multicolumn{14}{|c|}{ Sensitivity to inhibitory compounds } \\
\hline Crystal Violet $(0.5 \mu \mathrm{g} / \mathrm{ml})$ & - & - & - & - & - & - & - & - & - & + & - & + & - \\
\hline Phenol $(0.1 \%)$ & - & - & - & - & - & + & - & - & - & - & + & + & + \\
\hline \multicolumn{14}{|l|}{ (- Sensitive, + Resistant) } \\
\hline Growth at pH 4.5 & - & - & - & - & - & - & - & - & - & - & - & - & - \\
\hline Starch Hydrolysis & + & + & + & + & + & + & + & + & + & + & + & + & + \\
\hline Gelatin Liquification & Fast & - & - & Slow & - & - & - & Slow & Fast & - & Slow & Slow & Slow \\
\hline Nitrate reduction & + & + & + & + & + & + & + & + & + & + & + & + & + \\
\hline
\end{tabular}


Table.4 Identification of Streptomyces isolates

\begin{tabular}{|l|l|}
\hline Isolate & Identification \\
\hline $\mathrm{S}_{1}$ & Streptomyces scabies \\
\hline $\mathrm{S}_{2}$ & Streptomyces scabies \\
\hline $\mathrm{S}_{3}$ & Streptomyces spp. \\
\hline $\mathrm{S}_{4}$ & Streptomyces scabies \\
\hline $\mathrm{S}_{5}$ & Streptomyces aureofaciens \\
\hline $\mathrm{S}_{6}$ & Streptomyces aureofaciens \\
\hline $\mathrm{S}_{7}$ & Streptomyces spp. \\
\hline $\mathrm{S}_{8}$ & Streptomyces scabies \\
\hline $\mathrm{S}_{9}$ & Streptomyces scabies \\
\hline $\mathrm{S}_{10}$ & Streptomyces scabies \\
\hline $\mathrm{S}_{11}$ & Streptomyces scabies \\
\hline $\mathrm{S}_{12}$ & Streptomyces aureofaciens \\
\hline $\mathrm{S}_{13}$ & Streptomyces scabies \\
\hline
\end{tabular}

On the basis of aerial mycelium colour, spore chain morphology, vegetative mycelium colour (Table 1), growth on various media (Table 2), sugar utilization, sensitivity to antibiotics, melanin production, growth in inhibitory compounds and sensitivity at $\mathrm{pH}$ 4.5 (Table 3), isolates S1, S2, S4, S8, S9, S10, S11 and S13 with spiral chain morphology were categorized as Streptomyces scabies. Lambert and Loria (1989a) described some of the primary characters of Streptomyces scabies as smooth grey aerial mycelium borne in spiral spore chains, melanin production and utilization of all ISP sugars (Shirling and Gottlieb, 1966), sensitive to penicillin G $(100 \mu \mathrm{g} / \mathrm{ml})$, streptomycin $(20 \mu \mathrm{g} / \mathrm{ml})$, crystal violet $(0.5 \mu \mathrm{g} / \mathrm{ml})$ and inability to grow at $\mathrm{pH}$ 4.5 .

Eight spiral spore chain isolates (S1, S2, S4, S8, S9, S10, S11 and S13) were almost similar with each other. However, few differences were observed in connection with some sugar utilization. For instance, isolates S1, S2, S10, S11 and S13 utilized all sugars but isolate $\mathrm{S} 9$ could not utilized raffinose whereas isolate S4 could not utilized arabinose and raffinose. Isolate $\mathrm{S} 8$ was unable to utilize galactose and xylose. Isolates $\mathrm{S} 1$, S4, S8 and S9 were sensitive to streptomycin $(20 \mu \mathrm{g} / \mathrm{ml})$, penicillin $\mathrm{G}(100 \mu \mathrm{g} / \mathrm{ml})$, crystal violet $(0.5 \mu \mathrm{g} / \mathrm{ml})$ and phenol $(0.1 \%)$. The isolate S11 was sensitive Only to Streptomycin $(20 \mu \mathrm{g} / \mathrm{ml})$ and crystal violet $(0.5 \mu \mathrm{g} / \mathrm{ml})$. These physiological differences might be attributed to geographical variations of the test strains, as they were collected from different regions of Varanasi district. Elesawy (1979) also reported 452 variant strains of S. scabies, obtained from various diversified regions, differing in their morphological, cultural and physiological properties. On the basis of cultural and physiological properties, Sharma (1984) differentiated his isolates as three variant strains of Streptomyces scabies.

Flexuous spore chain isolates (S5, S6 and S12) showed almost all the primary morphological, cultural and physiological characteristics similar to Streptomyces aureofaciens as described by Faucher et al., (1992) which included bright yellow vegetative mycelium, turning brown after two weeks, grey aerial mycelium, flexuous spore chain, absence of melanin production, utilization of L-arabinose, D-fructose, Dglucose, D-mannitol, raffinose, sucrose and xylose, growth in the presence of Penicillin $\mathrm{G}$ $(100 \mu \mathrm{g} / \mathrm{ml})$ and $0.5 \%$ phenol, inhibition of growth by streptomycin $(20 \mu \mathrm{g} / \mathrm{ml})$ and inability to grow at $\mathrm{pH} 4.5$. 
Few variations were observed in connection with utilization of some sugars, reaction to antibiotics and inhibitory chemicals. Isolate S5 could not utilized Raffinose, whereas isolate S6 could not utilized Arabinose and Sucrose and isolate S12 could not utilized Galactose, Raffinose and Inositol. Here again, these differences may be attributed to the geographical variation of test strains, as they were collected from different locations.

Isolates S3 and S7 had morphological characters common with flexuous isolates but their cultural and physiological properties did not fall in any of the groups described earlier. Therefore, it has been considered as separate Streptomyces species. From the experimental findings, it can be stated that at least three different species of Streptomyces including Streptomyces scabies and Streptomyces aureofaciens are involved in causing common scab of potato in India (Table 4). Findings of Fischer et al., (2003) corroborate with our findings in that stated that Streptomyces species isolates with confirmed pathogenicity did not show antigens in common.

Out of the 27 isolates, only thirteen (48\%) were found to be pathogenic. This indicates that not only pathogenic ones but also saprophytic ones whose numbers may be more in the lesions of infected tubers. The same trend was also reported by Labruyere (1971) and Loria and Davis (1989).

\section{References}

Bonde, M.R. and McIntyre, G.A. 1968. Isolation and biology of Streptomyces spp. Causing Potato scab in soils below pH 5. Am. Potato J., 45: 273-278.

Cross, T. 1986. Growth and examination of actinomycetes- some guidelines. The Actinomycetes II in "Bergey's Manual of Systematic bacteriology", Pp. 23402343.
Elesawy, A.A. 1979. Isolation and characterization of $\mathrm{S}$. scabies strains from scab lesions of Potato tubers. Designation of the neotype strains of $\mathrm{S}$. scabies. Acta. Microbial. Acad. Sci. Hung., 26: 311-320.

Faucher, E., Savard, T. and Beaulien, C. 1992. Characterization of actinomycetes isolates from Common scab lesions on potato tubers. Can. J. Plant Pathol., 14: 197-202.

Fischer, K., Holt, D.C., Harumal, P., Currie, B.J., Walton, S.F. and Kemp, D.J. 2003. Generation and characterization of cDNA clones from Sarcoptes scabiei var. hominis for an expressed sequence tag library: identification of homologues of house dust mite Allergens. Am. J. Trop. Med. Hyg., 68: 61-64.

Healy, F.G. and Lambert, D.H. 1991. Relationship among Streptomyces spp. causing potato Scab. Int. J. Syst. Bacteriol., 41: 479-482.

King, R.R., Lawrance, C.H. and Calhoun, L.A. 1992. Chemistry of phytotoxins associated with Streptomyces scabies, the causal organism of potato common scab. J. Agri. Food Chem., 40: 834-837.

Kiraly, Z. 1974. Methods in plant pathology. Elsevier Scientific Pubs. Co., New York.

Labruyere, R.E. 1971. Common scab and its control in seed potato crop. Agric. Res. Report, (Verrl. Land Bouwk Onderz) 76 pp.

Lambert, D.H. and Loria, R. 1989a. S. scabies sp. Nov. Nam. Rev. Int. J. Syst. Bacteriol., 39: 387-392.

Lambert, D.H. and Loria, R. 1989b. S. acidiscabes sp. Nov. Int. J. Syst. Bacteriol., 39: 393-396.

Lawrance, C.H. 1956. A method of isolating actinomycetes from scabby potato tissue and soil with minimal contamination. Can. J. Bot., 34: 44-47. 
Lechevalier, M.P. 1988. Actinomycetes in agricultural and forestry. In: Goodfellow. M; Williams S.T. and Mordarski, M. (Eds.) Actinomycetes in Biotechnology, Academic Press, Sam Diego. pp. 327-358.

Loria, R. and Davis, J.R. 1989. Streptomyces scabies. In "N. W. Shaad, Ed. Laboratory guide for Identification of plant pathogenic bacteria." 2 nd eds, APS Press, St. Paul. 119 pp.

Nagaich, B.B. 1983. Disease resistance in potato in India, Indian Phytopath., 36: $1-10$.

Pridham, T.G. and Gottlieb, D. 1948. The utilization of carbon compounds by some Actinomycetes and aid for species determination. J. Bact., 56: 107-114.

Sharma, K.D. 1984. Studies on common scab of potato. Ph.D. Thesis: G. B. Pant Univ. Agriculture Technology, Pantnagar, Nainital (U.P.).

Shirling, E.B. and Gottlieb, D. 1966. Method for characterization of Streptomyces species. Int. J. Syst. Bact., 16: 313-340.

Szabo, I. and Marton, M. 1964. Comments on the first result of the International cooperative work on criteria used in characterization of Streptomycetes. Int. Bull. Bact. Nomen. Taxon, 14: 17-38.

\section{How to cite this article:}

Rohit Badaya and Srivastava, J.S. 2017. Physiological variability in Streptomyces spp. A causal organism of common scab of potato (Solanum tuberosum L.). Int.J.Curr.Microbiol.App.Sci. 6(6): 1104-1114. doi: https://doi.org/10.20546/ijcmas.2017.606.128 